\title{
Resiliencia del profesorado de Música chileno en el contexto de pandemia de COVID-19
}

Resilience of Chilean Music teachers in the context of COVID-19 pandemic

Raúl Jorquera Rossel

raul.jorquerar@userena.cl

Departamento de Música

Universidad de La Serena, Chile

ORCID: http://orcid.org/0000-0002-8470-4624

Ximena Valverde Ocariz ximena.valverde@gmail.com Annie Heuser Waldorfschule, Berlín, Alemania ORCID: http://orcid.org/0000-0001-9197-8044

Rodrigo Montes Anguita rodrigo.montes@upla.cl Facultad de Arte

Universidad de Playa Ancha, Chile ORCID: https://orcid.org/0000-0003-0328-0303

doi: 10.7203/LEEME.48.21695

Recibido: 29-09-2021 Aceptado: 22-11-2021. Contacto y correspondencia: Raúl Jorquera Rossel, Departamento de Música, Universidad de La Serena, Larraín Alcalde 2843, 1700000, La Serena, Chile.

\section{Resumen}

El presente artículo pretende dar a conocer cómo ha afectado el contexto de pandemia por COVID-19 las condiciones laborales y personales del profesorado de Música en Chile. Durante el primer año de esta pandemia, se consultó a 154 docentes de Música sobre su situación personal y profesional con el objetivo de conocer cómo estaban enfrentando el contexto educativo a distancia. Los datos recopilados y contrastados con la bibliografía consultada, permiten observar no solo una modificación y reinvención de sus prácticas educativas, sino además una importante capacidad de reinvención, de automotivación y de salir adelante. Estas capacidades se engloban en el concepto de resiliencia. Todo lo observado permite sentar las bases de una educación musical contextualizada y con la capacidad de adaptarse a la realidad y los continuos cambios que esta presenta, y donde el rol de la educación musical dentro del currículum se torna a su vez mas importante y significativo, tanto para el estudiantado, así como también para el propio desarrollo curricular del sistema educativo.

Palabras clave: Educación musical; estrés (académico); resiliencia (académica); adaptación de medios educativos.

\section{Abstract}

This article aims to show how the context of the COVID-19 pandemic has affected the working and personal conditions of music teachers in Chile. During the first year of this pandemic, 154 music teachers were consulted about their personal and professional situation in order to find out how they were dealing with the context of distance education. The data collected and contrasted with the bibliography consulted allow us to observe not only a modification and reinvention of their educational practices but also an important capacity for reinvention and self-motivation and an ability to move forward. These capacities are included in the concept of resilience. All of these observations lay the foundations for sensing a contextualized musical education with the ability to adapt to reality and the continuous changes that it presents and where the role of music education within the curriculum becomes in turn more important and significant, both for the student body as well as for the curriculum development of the educative system.

Key words: Music Education; Stress (Academic); Resilience (Academic); Educational Media Adaptation.

@Raúl Jorquera Rossel, Ximena Valverde Ocariz y Rodrigo Montes Anguita. The content of this article is the sole responsibility of the authors. The Revista Electrónica de LEEME and Universitat de València are not liable for any legal actions that may arise involving the article's content. Revista Electrónica de LEEME - Lista Electrónica Europea de Música en la Educación-. http://ojs.uv.es/index/php/LEEME/index ISSN: 1575-9563. Editores: Universidad de Valencia y Jesús Tejada. Visibilidad de esta revista: SCOPUS, Emerging Sources Citation Index (Clarivate), EBSCO, CINDOC (CSIC), Citefactor, COPAC, Dialnet, DICE (CSIC), DOAJ, e-revistas (CSIC), EBSCO Premier, ERIH+, Gale Cengage Learning, IN-RECS, IRESIE, LATINDEX, MIAR, OCLC Worldcat, RESH, REDIB, RILM Core Journals, SUDOC, ULRICHS. Esta revista es de acceso libre mediante licencia Creative Commons 4.0 CC by. Política de archivado: etiqueta verde SHERPA-ROMEO. 


\section{Introducción}

Durante el inicio del año 2020, los noticiarios de todo el mundo no dejaban de hablar de un virus que estaba afectando e incluso matando a muchas personas en la ciudad de Wuhan, China. A poco andar, las noticias llegaron desde Italia donde pueblos y ciudades iniciaban periodos de cuarentenas y en las siguientes semanas, esto se replicaba en la mayoría de los países europeos. Mientras aquello ocurría, Chile se encontraba en medio de la crisis social más grande de las últimas décadas. Múltiples demandas sociales, desigualdades estructurales, económicas y educacionales, llevaron al país a una profunda crisis que detonó el 18 de octubre de 2019. Este fenómeno se conocería luego como "estallido social". Desde esta fecha, el sistema educativo nacional vio afectada la regularidad de su funcionamiento por numerosos paros, protestas y huelgas generales a lo largo del país, las cuales fueron reprimidas con gran violencia por parte del estado, dejando como consecuencia numerosas víctimas mortales, además de heridos y mutilados oculares (Miranda et al., 2019; Heiss, 2020; Jimenez, 2020).

En medio de este turbulento contexto, se iniciaba en el país el año escolar 2020, pero tras solamente un par de semanas de clases y, en un hecho sin precedentes, las clases dejaron de ser presenciales y comenzaron a realizarse a distancia, debido al avance incontrolable de la pandemia tanto en el mundo como en Chile, donde la cifra de afectados aumentaba cada día. Se utilizaron para ello diferentes formatos de educación a distancia, todo de acuerdo a las posibilidades que tenían para entonces tanto las unidades educativas como, a su vez, el propio estudiantado a consecuencia de sus variadas realidades socioeconómicas.

Al comienzo, se pensó que esta situación se extendería por un tiempo acotado, pero se continuó con el trabajo educativo a distancia durante todo el año escolar. El año 2021 volvió a iniciarse académicamente de forma remota y recién durante el segundo semestre, en forma paulatina y gradual, se han vuelto a abrir los centros educativos de los diferentes niveles que conforman la educación formal chilena nacional, retomando poco a poco la modalidad presencial o, en algunos casos, híbrida.

En el marco de este contexto, surge esta investigación a partir de la problematización sobre el lugar que cumplía la educación musical, pero particularmente del rol que tenía el profesorado de Música y cómo se situaba en aquel momento. A través de un proceso de reflexión, surgieron las siguientes preguntas ¿Cómo afecta la calidad de vida y el estado emocional del profesorado la modalidad educativa virtual? y ¿De qué manera han salido adelante profesionalmente en este periodo las y los docentes de educación musical? Este fue el punto de partida para el desarrollo de la presente investigación, donde, a partir de estas preguntas, se decidió conocer la realidad del momento del profesorado de Música en Chile. 


\section{Marco teórico contextual}

En este apartado, se desarrollarán los conceptos abordados en la investigación como, a su vez, el contexto en el que se llevó a cabo.

\subsection{La educación musical en pandemia}

La aparición repentina de una emergencia sanitaria a escala mundial cambió los hábitos de vida de gran parte de la población de los países afectados. En el caso de Chile, el clima social estaba ya muy conmocionado por el estallido social iniciado en octubre de 2019 , creándose un clima de tensión e incertidumbre social que puso en entredicho la denominada "subjetividad neoliberal", entendida como "el pensar la sociedad según los principios de dicha ideología: individualismo, exaltación de los logros materiales, rechazo a lo comunitario" (Salinas et al., 2021). En el mes de abril de 2020, la totalidad de establecimientos educacionales en Chile se encontraban trabajando en un formato educativo a distancia, en sus diferentes, variadas y creativas modalidades de enseñanza, lo que afectaba a cerca de tres millones de estudiantes de los niveles de Educación Inicial, Primaria y Secundaria (Arriagada, 2020).

Comenzaron entonces nuevos e imprevisibles problemas para la continuidad del año académico. Por un lado, los problemas de conectividad de las propias instituciones educativas para las modalidades de educación virtual y, por otro lado, la falta de recursos y medios económicos que impedían a miles de estudiantes pertenecientes a familias que bordeaban o estaban dentro de la línea de la pobreza, tener acceso a la nueva modalidad educativa. Este contexto causó una alteración del estado emocional del profesorado, del alumnado y sus familias, los tres principales actores de la educación (Fernández et al., 2020).

A nivel del profesorado, se comenzaba a percibir el descontento que existía por la sobrecarga laboral, sumado a la continua minusvaloración de la educación musical, aún considerando que "Chile es considerado como excepción ante la dramática evidencia continental" (Angel-Alvarado y Lira-Cerda, 2017). Este descontento se habría incluso incrementado en el contexto educativo virtual (Rodríguez-Quiles, 2021). A lo descrito anteriormente, podemos incluir la falta de recursos para desarrollar las clases y, sobre todo, la falta de formación en herramientas educativas virtuales, situación que se hacía aún más patente entre el profesorado de mayor edad y que no utilizaba de forma regular nuevas tecnologías digitales en sus clases (Montoya, 2021). Fue así como se observaba una contradicción entre los llamados, tanto a nivel nacional como internacional, a la modernización y, sobre todo, a la digitalización de la educación escolar formal, disminuyendo cada vez mas la utilización del papel y el lápiz, siendo sustituidos por recursos digitales (Daniec y Torres, 2021). 
Teóricamente, estos llamados se presentaban como muy seductores para el oyente, sin embargo, la realidad económica y educacional del país no permitía que esto se desarrollara con facilidad. El desarrollo de estas ideas no fue consultado a quienes día a día realizaban la labor docente, ni tampoco se consideraron las reales necesidades del alumnado inmerso en el sistema escolar (Cisternas, 2021). Surgían entonces preguntas como: ¿el alumnado necesita realmente digitalizar sus aprendizajes con tanta premura, sobreponiéndose a la adquisición de otros saberes? Al término del primer semestre del año 2020, la educación formal en gran parte del mundo era impartida de forma remota.

\subsection{El profesorado de Música chileno durante la pandemia}

La realidad social, económica y emocional del profesorado no es en absoluto fácil, existe abundante literatura al respecto, ya que se trata de una problemática que no solamente afecta al profesorado en Chile, sino que también a educadoras y educadores de muchos países, incluso aquellos llamados del primer mundo (Barraca, 2010). El agobio laboral, un continuo estrés debido a la sobrecarga laboral, la falta de recursos y, sobre todo, los bajos ingresos económicos que recibe el profesorado en Chile, hace de esta profesión una elección poco atractiva para quienes pretenden ingresar a la universidad (Mardones, 2019). Las consecuencias de esta tormenta perfecta que se ha ido formando con los años muchas veces desemboca, por un lado, en docentes que abandonan el sistema educativo formal a los pocos años de haber ingresado (Equipo Elige Educar, 2021) y, por otro lado, en numerosas bajas laborales por salud, sobre todo a nivel socioemocional, como la depresión o el síndrome Burnout (Barraca, 2010), teniendo como consecuencia una importante sobrecarga emocional (Robinet-Serrano y Pérez-Azahuanche, 2020). Dentro de esta realidad, se encuentra el profesorado de Música en Chile, quienes, a pesar de aportar al desarrollo integral de sus estudiantes (Arredondo, 2019), debido a la baja carga horaria de la asignatura, se ven en la necesidad de trabajar en más de un centro educativo para acceder a una jornada laboral completa y reunir así los ingresos económicos necesarios para su subsistencia.

Como ya se expuso en el punto anterior, durante el año 2019 se vivió una revolución social en Chile y la educación no estuvo exenta de aquellos sucesos que marcaron la historia del país. De hecho, el fenómeno que provocó el estallido social fueron las demandas que el estudiantado llevó a la calle días previos. Como si lo anterior no fuese suficiente, con la llegada de la pandemia por Covid-19 a Chile, el sistema educativo nacional cambio prácticamente de un día para otro, sin espacios para una adecuada transición (Silas y Vázquez, 2020), poniendo a prueba las capacidades creativas del profesorado (Tunjo-Guerrero 2021).

Fue especialmente difícil dar continuidad a esta nueva modalidad debido a que existió una permanente presión del gobierno de Chile para retornar a la modalidad presencial, contraponiéndose a las evidencias del avance de la pandemia en el país. Finalmente, el año escolar 2020 terminó casi en su totalidad con clases vía telemática o en los diferentes formatos no

@Raúl Jorquera Rossel, Ximena Valverde Ocariz y Rodrigo Montes Anguita. The content of this article is the sole responsibility of the authors. The Revista Electrónica de LEEME and Universitat de València are not liable for any legal actions that may arise involving the article's content. Revista Electrónica de LEEME - Lista Electrónica Europea de Música en la Educación-. http://ojs.uv.es/index/php/LEEME/index ISSN: 1575-9563. Editores: Universidad de Valencia y Jesús Tejada. Visibilidad de esta revista: SCOPUS, Emerging Sources Citation Index (Clarivate), EBSCO, CINDOC (CSIC), Citefactor, COPAC, Dialnet, DICE (CSIC), DOAJ, e-revistas (CSIC), EBSCO Premier, ERIH+, Gale Cengage Learning, IN-RECS, IRESIE, LATINDEX, MIAR, OCLC Worldcat, RESH, REDIB, RILM Core Journals, SUDOC, ULRICHS. Esta revista es de acceso libre mediante licencia Creative Commons 4.0 CC by. Política de archivado: etiqueta verde SHERPA-ROMEO. 
presenciales, en gran parte, debido a la férrea resistencia del Colegio de Profesores de retornar a la presencialidad, poniendo siempre sobre la mesa las consecuencias sanitarias que conllevarían dicho retorno (Muñoz, 2020).

A lo anteriormente descrito, es importante agregar la urgencia de formar al futuro profesorado en competencias digitales que les permitan enfrentar de mejor manera contextos educativos virtuales (Domínguez-Lloria y Pino-Juste, 2021), entendiendo que problemáticas como las vividas en la actual pandemia por Covid-19 podrían repetirse en cualquier momento (Picón et al., 2021), sobre todo, si consideramos que el mundo que engloba los diversos sistemas educativos es digital (Dussel, 2020).

\subsection{La resiliencia}

Este artículo está centrado en la capacidad que tiene el profesorado de Música de sobreponerse a las continuas adversidades profesionales presentes en la educación formal chilena. A estas alturas no es desconocido para ningún integrante del sistema educativo el rol que tiene la educación musical en el currículum y cómo esta ha ido disminuyendo su presencia en la escuela actual, siendo sustituida por materias mal llamadas "importantes" (Valverde, 2018). La labor diaria del profesorado de Música al interior de las instituciones educativas no es fácil. En muchas ocasiones, no cuentan con los elementos mínimos necesarios para alcanzar los aprendizajes propuestos por las bases curriculares ministeriales (Valverde, 2013). Sin embargo, y a pesar de esto, el profesorado de Música continúa con su labor en el aula, muchas veces sin cuestionamientos, ya que es el momento en que pueden desarrollar y expresar todo su potencial pedagógico (Valverde, 2013).

Lo anteriormente descrito es vinculable con la capacidad humana conocida como resiliencia, que, según Benítez-Corona y Barrón-Tirado (2018), debe ser comprendida como una capacidad humana para vivir de forma positiva a pesar de la adversidad o situaciones difíciles que enfrentan las personas en los diferentes contextos sociales. Debido a sus características subjetivas, entenderla en profundidad puede resultar complejo. Por su parte, Grotberg (1996) define la resiliencia como la capacidad que tiene el ser humano para hacer frente a las adversidades de la vida. Yendo aún más allá, plantea que dicha capacidad no solo le hace frente a la adversidad, sino que, además, es capaz de superarlas e incluso puede llegar a transformarlas. Cuando se hace referencia a la resiliencia en el ámbito de la educación, es necesario comprenderla como una especie de resorte moral que ayuda a la persona (docente, estudiante) a no dejarse abatir y ser capaz de superar la adversidad (Noriega et al., 2015).

Para Noriega et al. (2015), la resiliencia en la educación contiene los llamados factores de riesgo que serán aquellos que tengan la posibilidad de causar algún tipo de daño a la persona afectada. Podemos ejemplificar esta definición en el ámbito de la educación musical: el 
profesorado de Música de una institución no cuenta con los elementos mínimos para desarrollar su clase, no cuenta con instrumentos musicales, ni tampoco con un espacio adecuado para realizar su labor educativa de forma correcta. Pero, por otro lado, existen los factores protectores que son aquellas condiciones que favorecen el desarrollo de las personas y reducen los efectos desfavorables. El profesorado, pese a no tener los recursos mínimos para el trabajo, ha sido capaz de formar agrupaciones musicales dentro de la unidad educativa y ha conseguido que los niños y niñas se motiven, logrando con este accionar que el colegio los empiece a considerar (Valverde y Godall, 2018). De esta forma, la resiliencia es vista como un proceso dinámico que depende de factores internos (personales) y externos (contextuales), tanto de riesgo como protectores. Ambos factores interactúan entre sí para generar un mecanismo que posibilita el desarrollo de algunos aspectos a pesar de las circunstancias, lo que se entiende como una adaptación positiva (Morelato, 2011; 2014).

Para Román et al. (2020), el termino resiliencia comprende, a su vez, dos variaciones del mismo. El primero se refiere la "resiliencia clásica", entendida como la capacidad de una persona o grupo para afrontar y sobreponerse a las adversidades y la segunda la "resiliencia generativa", la que se asocia a la capacidad de generación de alternativas y cambios para seguir adelante. Para Fiorentino (2008), se hace necesario que el profesorado cuente con una mayor capacidad de ser resiliente y de esta forma desenvolverse de mejor forma dentro del sistema educativo, lo que permitiría además aminorar los efectos negativos generados por las complicaciones del contexto (Robinet-Serrano y Pérez-Azahuanche, 2020).

\section{Marco metodológico}

El presente artículo es parte de una investigación de mayor alcance en la que se utilizó un enfoque mixto. No obstante, los resultados seleccionados para ser presentados en este artículo son analizados desde un enfoque metodológico cualitativo. No se plantearon hipótesis y se utilizaron preguntas de investigación que fueron respondidas a partir del análisis de los datos. Estas interrogantes de investigación, expuestas en la introducción, son las siguientes ¿Cómo afecta la calidad de vida y el estado emocional del profesorado la modalidad educativa virtual? y ¿De qué manera han salido adelante profesionalmente en este periodo las y los docentes de educación musical?

Esta investigación se basa en una metodología inductiva, es por esto que se apoya en principios de la Teoría Fundamentada, debido a sus características emergentes específicas que contribuyen a otorgar una mirada interpretativa de los datos recogidos (Strauss y Corbin, 2002), permitiendo dar un sentido narrativo a la información emergente de dicho discurso (Charmaz, 2004).

@Raúl Jorquera Rossel, Ximena Valverde Ocariz y Rodrigo Montes Anguita. The content of this article is the sole responsibility of the authors. The Revista Electrónica de LEEME and Universitat de València are not liable for any legal actions that may arise involving the article's content. Revista Electrónica de LEEME - Lista Electrónica Europea de Música en la Educación-. http://ojs.uv.es/index/php/LEEME/index ISSN: 1575-9563. Editores: Universidad de Valencia y Jesús Tejada. Visibilidad de esta revista: SCOPUS, Emerging Sources Citation Index (Clarivate), EBSCO, CINDOC (CSIC), Citefactor, COPAC, Dialnet, DICE (CSIC), DOAJ, e-revistas (CSIC), EBSCO Premier, ERIH+, Gale Cengage Learning, IN-RECS, IRESIE, LATINDEX, MIAR, OCLC Worldcat, RESH, REDIB, RILM Core Journals, SUDOC, ULRICHS. Esta revista es de acceso libre mediante licencia Creative Commons 4.0 CC by. Política de archivado: etiqueta verde SHERPA-ROMEO. 
Se procedió a codificar, desde una perspectiva inductiva, las unidades de significado emergentes del discurso de los/as participantes, conformando así pre-categorías que dieron pie a la conformación de dos bloques categoriales.

\subsection{Instrumentos de recolección de la información}

La recolección de la información para esta investigación estuvo dividida en dos etapas. En primer lugar, se comenzó con un análisis documental y, en segundo lugar, un cuestionario. Para Ruiz (2012), el análisis documental se enmarca dentro de las ciencias de la documentación y tiene como objetivo la necesidad de extractar la información necesaria y relevante de las fuentes documentales primarias de investigación. Por su parte, Del Rincón et al. (1995) organiza el análisis documental en tres fases: fase de entrada que, en el caso de este estudio, no tuvo inconvenientes ya que la información recopilada se basó en revistas científicas de divulgación en formato online de acceso abierto. Se siguió con la fase de reelaboración, que se enfocó específicamente en el análisis profundo de dicha documentación para dar paso a la fase de salida, que, para los términos de esta investigación, correspondió a la fundamentación teórica y al análisis de los datos obtenidos.

Rodríguez et al. (1996) consideran que el cuestionario es una forma de encuesta, pero caracterizada por la ausencia del encuestador. Además, indican que se trata un procedimiento exploratorio tanto de ideas como de creencias de algún aspecto de la realidad, en nuestro caso, sobre la realidad del profesorado de educación musical de Chile durante la pandemia. Entre los aspectos positivos de este instrumento, se reconoce que su administración no produce rechazo alguno entre los miembros de determinado colectivo, sino que es mayoritariamente aceptado. Por esta razón, se le considera una técnica útil en el proceso de acercamiento a la realidad estudiada.

La elaboración del instrumento, siguiendo a López-Roldán y Fachelli (2015), se realizó a través de los siguientes pasos: formulación, prueba piloto para su validación, revisión y ajustes para concluir con su difusión y aplicación. La prueba piloto fue respondida por siete docentes, quienes no participaron a posterior en la encuesta definitiva. Estos completaron, además, una pauta de evaluación en la que registraron sugerencias que fueron consideradas en la versión final del instrumento.

El instrumento fue difundido a través de redes sociales y correos electrónicos seleccionados de bases de datos de docentes de Música a las que se tuvo acceso. Este estuvo disponible para responder durante los meses de julio a agosto de 2020. Las respuestas fueron anónimas y no se consideraron ni los años de trabajo de los participantes, ni la región (provincia) a la cual pertenecían. 
Tabla 1. Resumen del instrumento

\begin{tabular}{clccc}
\hline Ítem & \multicolumn{1}{c}{ Descripción } & Cantidad de preguntas & Carácter \\
\hline I & Datos personales de quienes participan & 4 & Respuestas obligatorias \\
II & Trabajo educativo a distancia & 6 & Respuestas obligatorias \\
III & $\begin{array}{l}\text { Fortalezas y debilidades de la } \\
\text { educación a distancia en el contexto }\end{array}$ & 9 & Respuestas obligatorias y \\
& actual & & la última de carácter \\
& & & voluntario \\
\hline
\end{tabular}

Ahora bien, el centro de la información abordada en el presente artículo gira en torno al análisis del discurso de las respuestas a la pregunta abierta de la encuesta, pregunta en la que se les propuso a las personas participantes desarrollar con mayor profundidad su percepción en torno a la problemática abordada en el cuestionario, la pregunta fue la siguiente: ¿Podrías compartir un breve comentario de lo que ha significado a nivel personal y profesional el actual formato de educación a distancia? El análisis de discurso se ha constituido en una herramienta fundamental a la hora de valorar epistémicamente el lenguaje, observado no solamente como un vehículo de expresión de ideas, sino además como un factor que participa y tiene injerencia en la construcción de la realidad social (Santander, 2011).

\subsection{Participantes}

La muestra incluyó a 154 participantes, quienes respondieron la encuesta de manera telemática. El principal criterio de selección fue establecido en el instrumento y sus convocatorias: ser docentes de educación musical que se encontrasen trabajando durante el año 2020, ya sea en el contexto formal como en el no formal. Los/as participantes se encontraban trabajando, en su mayoría en el sistema de educación formal. En este contexto, la mayoría se desempañaba en Enseñanza Primaria, en segundo lugar, en Enseñanza Secundaria y, en tercer lugar, en Educación Inicial, además de participantes que desarrollaban su labor educativa en Educación Superior y, en otros casos, en talleres orquestales o instrumentales de academias y/o escuela de Música, como se observa en la Figura 1.

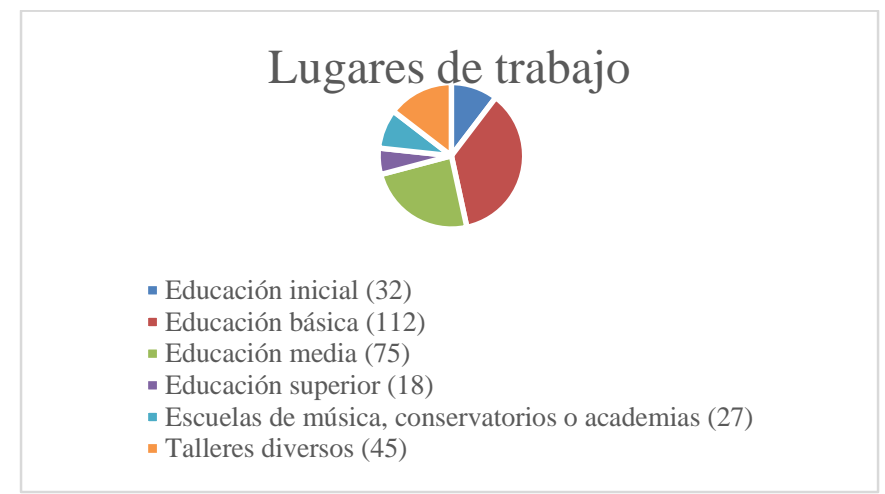

Figura 1. Lugares de trabajo en los que el profesorado se desempeñaba 
En relación al tipo de institución en que trabajaba el profesorado participante, la mayoría desarrollaba sus funciones en establecimientos particulares subvencionados $\mathrm{y}$, en segundo lugar, instituciones educativas de carácter municipal (Figura 2).

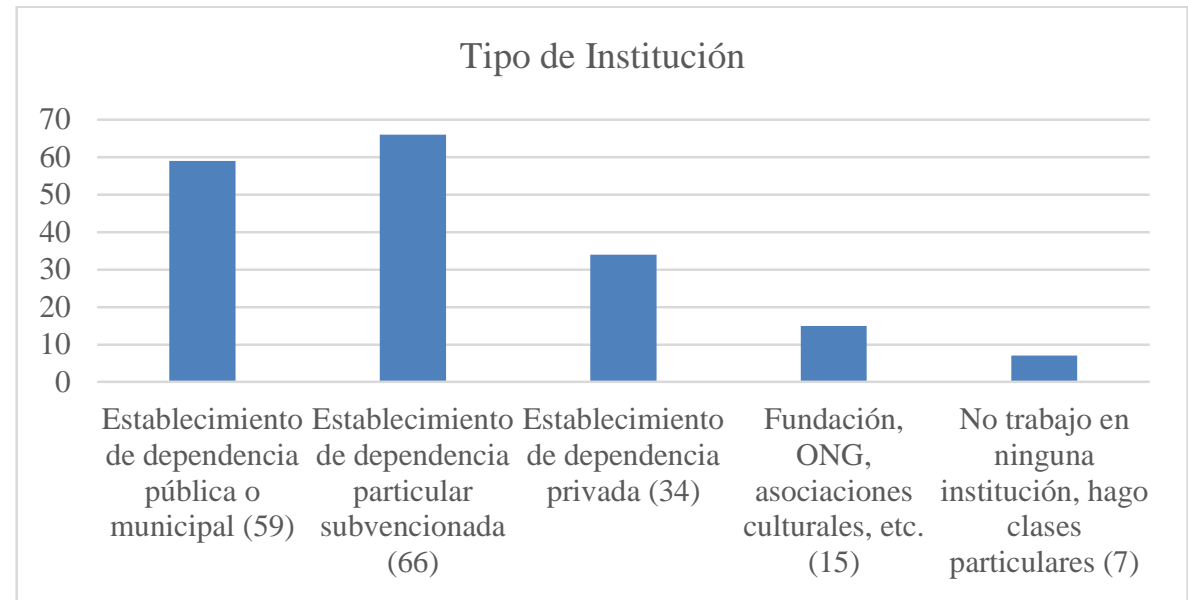

Figura 2. Tipo de institución en la que se desempeñaba el profesorado participante

En la caracterización de los/as participantes, fue importante conocer en qué contexto desarrollaban sus clases, entendiéndose este como contexto urbano o rural. 136 participantes se encontraban trabajando en el contexto urbano, se contó con las respuestas de 6 docentes que se desempeñaban exclusivamente en el contexto rural y, además, 5 docentes que trabajaban en ambos contextos, urbano y rural (Figura 3).

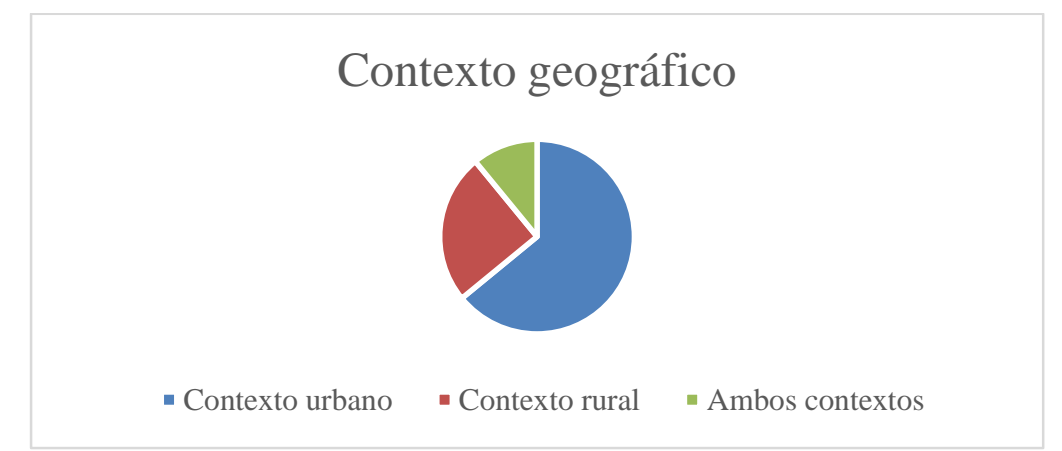

Figura 3. Contexto geográfico en el que se desempeñaba el profesorado participante

\subsection{Categorías de significado}

Para este ámbito del estudio, vinculado a las respuestas a la pregunta abierta de la encuesta, se optó seguir los lineamientos de la Teoría Fundamentada, debido a su apertura a observar el discurso aportado por las personas participantes desde un enfoque interpretativo,

@Raúl Jorquera Rossel, Ximena Valverde Ocariz y Rodrigo Montes Anguita. The content of this article is the sole responsibility of the authors. The Revista Electrónica de LEEME and Universitat de València are not liable for any legal actions that may arise involving the article's content. Revista Electrónica de LEEME - Lista Electrónica Europea de Música en la Educación-. http://ojs.uv.es/index/php/LEEME/index ISSN: 1575-9563. Editores: Universidad de Valencia y Jesús Tejada. Visibilidad de esta revista: SCOPUS, Emerging Sources Citation Index (Clarivate), EBSCO, CINDOC (CSIC), Citefactor, COPAC, Dialnet, DICE (CSIC), DOAJ, e-revistas (CSIC), EBSCO Premier, ERIH+, Gale Cengage Learning, IN-RECS, IRESIE, LATINDEX, MIAR, OCLC Worldcat, RESH, REDIB, RILM Core Journals, SUDOC, ULRICHS. Esta revista es de acceso libre mediante licencia Creative Commons 4.0 CC by. Política de archivado: etiqueta verde SHERPA-ROMEO. 
permitiendo dar un sentido narrativo a la información emergente de dicho discurso (Charmaz, 2004).

Se procedió a codificar, desde una perspectiva inductiva, las unidades de significado emergentes del discurso de los/as participantes, conformando así pre-categorías que dieron pie a la conformación de dos bloques categoriales, el primero denominado "Estrés", con sus subcategorías "Agobio personal y familiar" y "Agobio profesional y laboral". El segundo bloque fue el denominado "Resiliencia" con las correspondientes categorías "Aceptación de desafíos" y "Nuevas herramientas educativas".

\section{Resultados}

En los siguientes apartados, se desarrolla el análisis a partir de cada uno de los bloques categoriales y sus correspondientes subcategorías. Siguiendo las propuestas de la Teoría Fundamentada, se busca articular el análisis del discurso de los participantes, a través de unidades de significado, con la conceptualización desarrollada en el marco teórico del estudio.

De las unidades de significado seleccionadas para el análisis surgen de manera esporádica conceptos que probablemente no sean del todo comprendidos en un contexto de español internacional, por lo que en estos casos se ha optado por incluir entre paréntesis sinónimos para cada uno de ellos.

\subsection{Estrés: agobio personal y familiar}

El concepto de estrés surge de manera explícita en el discurso del profesorado participante. Se trata de un estrés vinculado al tiempo extra que se debe dedicar al ámbito laboral: "Trabajo más y la crisis aumenta el estrés" (d.27). En esta misma línea se pudo observar, incluso, catarsis genéricas que evidencian la sensación de malestar global frente al contexto de educación a distancia, como este ejemplo: "Solo diré: Estrés total y completo caos" (d.71), o bien “...caótico y confuso, lleno de dudas" (d.58). Este estrés observado en las unidades de significado aportadas por las y los docentes participantes se relaciona con otras dimensiones, como es el caso del agotamiento físico: "Ha sido una experiencia muy estresante y agotadora, considerando que debo hacer clases con mi hijo de dos años junto a mí" (d.22). Ademas, vinculado al estrés, se observan segmentos que reflejan sensaciones de frustración entre los docentes participantes: "Siento frustración y mucha pena" (d.8).

El cansancio, el agotamiento y el desgaste emergen como elementos recurrentes cuando se analiza la narración del profesorado de la especialidad participante en el estudio: "Mucho desgaste en reuniones, clases virtuales, preparación de material y revisión de trabajos" (d.131). El agobio generado por el trabajo frente a la pantalla de los dispositivos con los que el profesorado 
participante se asocia a una pérdida de espacios personales: "Espacio personal invadido, mayor cansancio por la cantidad de horas frente al computador" (d.23) y, en voz del mismo profesorado participante, una inversión mayor de tiempo frente a la planificación y evaluación del proceso educativo: “...no hay tiempo libre, ocupa mucho más tiempo en preparación de clases y revisión de tareas" (d.23), o bien "Me siento colapsada, no tengo tiempo para mi" (d.153).

Este espacio personal invadido afecta, según el discurso de una de las docentes participantes, no solo el ámbito personal, sino que, de manera directa, a las relaciones intrafamiliares: "Mi tiempo personal y familiar se ve afectado" (d.14). Esta problemática conlleva tensiones en el intento de conciliar la dimensión laboral con los diversos ámbitos que configuran la dimensión personal de quienes participaron en este estudio, como lo menciona la siguiente docente: "A nivel personal me afecta, ya que soy mamá de una niña de dos años y es agotador conciliar la crianza, las labores domésticas, con el trabajo de docente" (d.6). Las tensiones intrafamiliares generadas, como producto de la mayor dedicación temporal por parte de quienes participaron en este estudio afectarían además al resto de integrantes de los grupos familiares, conflicto que queda muy bien reflejado en el discurso de una de las profesoras: "Ha sido un desgaste emocional para mis hijos ya que, por estar conectada durante el día, no puedo prestar atención a sus actividades" (d.95). A su vez, este conflicto se amplía al terreno de los espacios de trabajo familiares:

"En el ámbito personal, fue caótico en un comienzo, ya que compartíamos computador con mi hermano (con clases on-line todo el día), y yo solo podía trabajar en la noche, lo que fue sumamente agotador a nivel emocional, mental y también físico" (d.30).

Todos estos hallazgos están en concordancia con los estudios de Fernández et al., 2020; Picón et al., 2021; Robinet-Serrano y Pérez-Azahuanche, 2020 y Román et al., 2020.

\subsection{Estrés: agobio profesional y laboral}

El agobio personal familiar analizado en el apartado anterior se relaciona, necesariamente, con un agobio profesional y laboral. Al explorar el discurso de quienes participaron en este estudio, surgen de manera espontánea una serie de unidades de significado que estructuran esta subcategoría. Este agobio se vincula de manera natural con la observación que hacen las y los docentes sobre el estudiantado que participa en sus clases bajo la modalidad educativa analizada: "Sólo estrés, así lo manifiestan también los estudiantes, las horas frente al PC (ordenador), está costando muchos síntomas de estrés y depresión, profesoras llorando la presión. No, todo mal” (d.150).

La sensación de no estar cumpliendo de buena manera el rol docente encomendado, traducido en una insatisfacción laboral, emerge como una de las variantes del bloque categorial estrés, tal como lo expresa el siguiente docente: "Siento que podría hacer mejor mi trabajo y esa 
sensación me invade todo el tiempo" (d.59). Esta sensación, en determinados casos, se manifiesta a modo de incertidumbre: "Es un trabajo incierto, no sabes si lo qué haces está bien" (d.75). Esta insatisfacción laboral incluso puede representar un factor que afecta la relación emocional de esta docente participante con su ejercicio profesional: "poder disfrutar de tu docencia se hace lejano por sentir que le faltas a tus estudiantes" (d.111).

La relación emocional del profesorado con su desempeño profesional se ve afectada también por las características educativas del contexto. Eso queda de manifiesto cuando se incluye en el discurso la participación del estudiantado en las actividades lectivas virtuales: "La incertidumbre en el futuro, el encierro, la calidad de la comunicación y respuesta de los alumnos generan un nivel de ansiedad considerable" (d.152). Ahora bien, en el marco de la variante emocional de esta subcategoría, es relevante identificar las implicancias desde la especialidad artístico-musical: "Me ha afectado mucho no tener el contacto físico, la interacción en vivo, el saludo, un abrazo, extraño el tocar juntos" (d.144). La imposibilidad de hacer música en conjunto es uno de los principales factores que estarían afectando al profesorado de la especialidad, como lo ejemplifica el discurso de la siguiente profesional participante: "Muy estresante y cansador. En las artes, las sensaciones, emociones, la expresión musical no se reflejan a través de la pantalla. La retroalimentación y motivación sin estar cerca es casi imposible...no me adapto" (d.116).

Si centramos el análisis en las características específicas del trabajo educativo virtual, surgen factores que incrementan los niveles de estrés y agobio profesional en el profesorado participante, como es lo vinculado a la entrega de trabajos por parte del estudiantado: "Las tareas (deberes) han aumentado mucho en esta nueva modalidad, lo que me mantiene con un nivel de estrés mayor de lo común" (d.69), idea reforzada por lo expuesto por el siguiente docente: "Personalmente la carga de trabajo ha sido duplicada, mucho de ese trabajo es solo administrativo y no veo en él aporte al aprendizaje de mis estudiantes" (d.57).

La preocupación por el aprendizaje del alumnado surge como un factor recurrente cuando analizamos el agobio profesional y laboral en el discurso del profesorado participante en el estudio. En algunos casos, esta preocupación se relaciona con las características de los niveles con los que interactúan educativamente, tal como lo expone la siguiente docente participante:

\footnotetext{
"A nivel profesional es complicada la educación a distancia ya que el trabajo en los niveles en los cuales me desarrollo (pre-básica y básica), es más práctico y no tan teórico, por lo que las actividades de práctica vocal, actividades corporales, rítmicas y de audición se ven afectadas por internet" (d.6).
}

Esta inquietud también apunta en forma directa a la modalidad virtual, en lo específico a las opciones sincrónicas o asincrónicas que ofrecen las diversas plataformas de trabajo educativo a distancia y las expectativas que estas generaban en el profesorado, tal como lo expresa el siguiente docente participante en el estudio: 
"En un principio, confiaba en que el actual formato de clases a través de Classroom y Meet ayudaría a desarrollar de buena manera la asignatura, pero esto requería de una motivación previa por parte de los estudiantes hacia el aprendizaje y con el tiempo me he dado cuenta que en general esto no existe" (d.154).

Siempre en el ámbito de la subcategoría de agobio profesional y laboral, otra variante presente en las unidades de significado extractadas del discurso del profesorado participante guarda relación con el estrés generado al verse obligados a utilizar herramientas de tecnología educativa desconocidas por este colectivo, tal como lo narra la siguiente docente: "Aprender muchas estrategias nuevas en tecnología educativa y me he estresado mucho" (d.68), planteamiento que es reforzado por el siguiente docente: "Ha sido difícil porque no estaba preparada para esta nueva normalidad a distancia, me faltan recursos y conocimiento en edición de audio y video" (d.82). Quienes debieron invertir más tiempo personal en su proceso de autoperfeccionamiento manifiestan su malestar frente a esta situación, como es el caso del siguiente docente: "Ha sido agotador, en el sentido que, a la vez que preparas tus clases, también debes perfeccionarte en herramientas y programas digitales, duplicando, así el tiempo de trabajo diario" (d.39).

Los resultados descritos en este apartado concuerdan con lo descrito por: Daniec y Torres, (2021), Fernández et al. (2020), Montoya (2021), Robinet-Serrano y Pérez-Azahuanche (2020) y Tunjo-Guerrero (2021).

\subsection{Resiliencia: nuevas herramientas educativas}

Al explorar la narrativa del profesorado participante en esta investigación, no solo es posible observar unidades de significado vinculables al estrés y al agobio tanto personal como profesional, sino que surgen además segmentos que dan cuenta de posiciones que pueden ser vinculadas a la resiliencia clásica, reflejando tendencias a adaptarse a las dificultades contextuales desde las exigencias tecnológicas del formato educativo virtual, tal como lo afirma el siguiente docente: "Creo que es una buena herramienta que nos quedará a futuro para apoyar las clases presenciales. He tenido que aprender cosas nuevas" (d.14), posición reforzada por lo expuesto por el siguiente docente participante: "He tenido la oportunidad de aprender a utilizar múltiples herramientas tecnológicas que han servido para replantear metodologías y actividades con mis estudiantes" (d.151). Por otro lado, vinculado a la adaptación a nuevas herramientas educativas, es posible apreciar unidades de significado que evidencian cómo esta adaptación se produce al disponer de una mayor cantidad de tiempo para poder lograrlo: "He tenido más tiempo para dedicarme a utilizar las tecnologías y redes sociales en la didáctica musical" (d.19). Esta adaptación a nuevas herramientas educativas permite que a través de la creatividad puedan emerger estas nuevas alternativas metodológicas: 
"A nivel personal, he tenido urgentemente que adaptarme a tecnologías que hace tres meses no usaba, como la edición de audio y video, así como utilizar metodologías que considero más entretenidas para mis estudiantes como títeres, repertorio más lúdico y mayor creatividad en los guiones de mis clases" (d.134).

Al continuar el análisis del discurso del profesorado participante, emergen segmentos que reflejan un vínculo en la adaptación a la modalidad educativa virtual con la relación docenteestudiante, relación que, desde una perspectiva resiliente, podría ser reenfocada gracias a la modalidad: "Me ha dado la oportunidad de establecer otras conexiones con los estudiantes y los apoderados. Aprender a utilizar diversas aplicaciones y herramientas que propician esas conexiones" (d.41). Ahora bien, esta adaptación a la modalidad educativa online es vinculable con la búsqueda de nuevas estrategias docentes: "Ha mejorado el interés y las capacidades para aprender de nuevas estrategias educativas" (d.29). Estas nuevas estrategias podrían además aportar a la motivación por parte del alumnado: "Posibilidades de explorar otras formas de educar, apuntando aún más a la motivación" (d.72).

Ahora bien, el relato que apunta a la opción de repensar las estrategias educativas, como producto de esta adaptación al formato educativo virtual, puede ser contrapuesto por lo expuesto por la siguiente docente, quien si bien observa como algo positivo la oportunidad de aplicar nuevas estrategias docentes, establece una crítica desde su visión particular en contra del procedimiento adoptado, en el mismo marco contextual, por sus pares docentes: "Ha significado una oportunidad de poner en práctica ideas nuevas que uno tiene, aunque creo que también ha sacado a la vista la poca creatividad de algunos colegas" (d.83).

La oportunidad que ofrece el contexto para acercarse a nuevas herramientas y estrategias educativas, en algunos casos, es vista como una alternativa para implementar ideas y proyectos que en un contexto pre-pandémico hubiesen tardado en hacerse efectivos, tal como lo narra el siguiente docente que participó en el estudio:

"He podido desarrollar proyectos que tenía esperando poder realizar a este nivel, de interacción virtual y utilización de las herramientas respectivas en el ámbito de la enseñanza musical y, a la vez, proyectando nuevos formatos para las clases" (d.87).

Los datos expuestos en este apartado son consistentes con los estudios presentados por: Benítez-Corona y Barrón-Tirado (2018), Fiorentino (2008), Morelato (2011; 2014), Noriega et al. (2015), Robinet-Serrano y Pérez-Azahuanche (2020) y Román et al. (2020).

\subsection{Resiliencia: aceptación de desafíos}

Cuando se analiza el discurso del profesorado participante en el estudio, surgen una serie de narraciones que dan cuenta de una capacidad de sobreponerse a la problemática del contexto, visualizando las adversidades halladas como oportunidades de crecimiento: "Sin duda que presenta una oportunidad de crecimiento toda esta situación" (d.41). Estas posiciones se enmarcan 
dentro del concepto de resiliencia generativa, sobre todo, si se refuerza con la posición de otros docentes participantes, quienes dimensionan la adversidad del contexto como un desafío que aporta a un crecimiento personal: "Solo queda verlo como un desafío del cual todos podemos aprender" (d.89), "Hay que adaptarse y tratar de buscar herramientas nuevas y ver nuevas oportunidades en lo que podría ser un problema" (d.81) o bien "Un desafío personal en cuanto a la adaptación a una nueva modalidad de trabajo, así como de vida" (d.147).

Enmarcados en la resiliencia clásica, se observan relatos que vinculan las oportunidades de crecimiento personal con las opciones educativas que surgen de las adversidades contextuales: "Gratificante, ya que me he puesto a prueba y tengo la capacidad de buscar lo mejor en circunstancias adversas, aprendido formas nuevas de enseñar música" (d.124), relato que es reforzado por la siguiente docente participante, quien incluye, además, el concepto creatividad: "Ha significado poner a prueba la creatividad, abrir la mente y explorar nuevos formatos, lo cual es bastante gratificante" (d.126).

La aceptación de nuevos desafíos emerge en el discurso del profesorado participante como un relato resiliente, el que se vincula, en algunos casos, al ámbito emocional y su relación con la dimensión educativa: "Pienso que la contención y el transmitir tranquilidad han pasado a un primer plano en este periodo, relegando el contenido de la asignatura propiamente tal a un segundo plano" (d.76). Posición que apunta a entender las adversidades de la contingencia como una oportunidad de establecer un giro en el sistema educativo: "Desafío de enfrentar un nuevo sistema de enseñanza" (d.3), idea reforzada por otros docentes participantes: "Representa un desafío tremendo, pero a la vez necesario. Obliga a replantearse el escenario educativo en dimensiones no anteriormente previstas" (d.20), "Ha sido un proceso de resignificación de la enseñanza y de la propia asignatura" (d.60) o bien "Un rompimiento paradigmático, desde el punto de vista didáctico y metodológico... hay que repensar lo esencial en la educación" (d.80).

En esta narrativa enfocada desde el enfoque de la resiliencia generativa (Román et al., 2020), en algunos segmentos analizados del discurso del profesorado participante, se puede observar posiciones que, incluso, van más allá de una simple aceptación de los desafíos contextuales, sino que reflejan una completa adaptación a los nuevos formatos educativos:

"Ha significado por sobre todo un aprendizaje, me he motivado con esta nueva forma de trabajo, quizás en estos momentos me cueste más volver a la forma normal de trabajo pues luego de un gran esfuerzo, me acomodé a esto" (d.36).

La información presentada en este apartado concuerda con los trabajos de Benítez-Corona y Barrón-Tirado (2018), Fiorentino (2008), Morelato (2011; 2014) Noriega et al. (2015), RobinetSerrano y Pérez-Azahuanche (2020) y Román et al. (2020). 


\section{Conclusiones}

Sin duda alguna, la modalidad educativa a distancia ha sido uno de los grandes desafíos para los diversos sistemas educacionales a nivel internacional. Es en esta dimensión en la que el profesorado ha debido sostener el engranaje en el que convergen, por un lado, las necesidades del estudiantado junto a sus familias y, por otro lado, los requerimientos de la administración de los diversos centros educativos en conjunto a las exigencias del currículum nacional (Dussel, 2020).

De la información aportada por los/as participantes, no se observan diferencias relevantes entre su discurso y el tipo de institución en el que se desempeñaban, el nivel educativo en el que desarrollaban su función docente ni el contexto geográfico en el cual llevaban a cabo su labor educativa, situación que permitiría considerar que tanto las percepciones de agobio como las actitudes resilientes identificadas serían de carácter transversal.

Frente a la primera pregunta de investigación, ¿Cómo afecta la calidad de vida y el estado emocional del profesorado la modalidad educativa virtual?, es posible observar en el discurso del profesorado participante en el estudio que el contexto educativo a distancia se constituyó en un primer momento como un desafío complejo de superar. En este contexto, estuvieron presentes de manera recurrente sensaciones de agobio, agotamiento físico y mental, reacciones vinculadas al estrés, confusión e incertidumbre, además de sentir una invasión de espacios y tiempos, tanto personales como familiares, todo esto generado por una sobrecarga emocional (Picón et al., 2021; Román et al., 2020).

Este agobio anteriormente descrito podría ser aún mayor si se considera que el profesorado de la especialidad partícipe en el estudio se encontraba aún asimilando las consecuencias emocionales, tanto personales como educativas, de la crisis social presente en el país, la que tuvo como punto máximo el denominado estallido social, instancia que remeció los cimientos, no solo del sistema educativo nacional, sino también de la sociedad chilena en su conjunto (Olave et al., 2020; Salinas et al., 2021; Silva-Peña y Paz-Maldonado, 2019).

El profesorado de música chileno, desde una perspectiva histórica, ha sabido sobreponerse a una continua y creciente minusvaloración de la asignatura (Arredondo, 2020; Rodríguez-Quiles, 2021), situación que permitiría sostener la existencia de unas posiciones y actitudes resilientes previas, las que habrían permitido sobreponerse de una mejor manera a las dificultades generadas por el contexto educativo a distancia. Esta resiliencia podría incluso llegar a disminuir los efectos de las situaciones estresantes descritas. Esta posición observada en el discurso del profesorado partícipe del estudio, vinculada a la resiliencia clásica (Román et al., 2020), se manifiesta en una aceptación del gran desafío que fue comenzar el año académico 2020 en una modalidad nunca antes trabajada, utilizar herramientas y plataformas virtuales que en muchos casos eran desconocidas, enfrentar este desafío como una oportunidad de crecimiento 
personal y una profundización de sus capacidades de adaptación. Es relevante destacar aquí la urgencia de incrementar competencias digitales en los programas de formación docente del profesorado para enfrentar de mejor manera contextos similares futuros (Domínguez-Lloria y Pino-Juste, 2021).

Si consideramos la segunda pregunta de investigación, ¿De qué manera han salido adelante profesionalmente en este periodo las y los docentes de educación musical?, se observa que el profesorado del estudio percibió las dificultades del contexto como una oportunidad de acercarse a una diversidad de herramientas tecnológico-educativas, acercamiento mediado por una autocapacitación en el uso de estas herramientas y plataformas. A su vez, desde una prospectiva resiliente generativa (Román et al., 2020), el contexto educativo virtual fue considerado como un espacio para poner en práctica nuevas estrategias didácticas y proyectos que no habían sido ser ejecutados en el devenir de la "normalidad" educativa pre-pandémica.

Todo lo anterior refleja que el profesorado de Música que participó en el estudio muestra una disposición a desarrollar una educación musical focalizada en la formación integral (Arredondo, 2020) y contextualizada, desde un enfoque reflexivo y, por qué no decirlo, orientado a profundizar un pensamiento libre, crítico y autónomo (Jorquera et al., 2020). Esta disposición del profesorado estaría en tensión con las políticas ministeriales para la asignatura, las que, al tener un carácter nacional, consideran de manera muy débil las diferencias territoriales y contextuales en las que desarrollan el trabajo educativo del profesorado del país, problemática que se pudo haber profundizado en el contexto educativo virtual, modalidad educativa impuesta en el marco de las medidas sanitarias generadas por la pandemia por COVID-19.

La principal limitación observada en este estudio se relaciona con los posibles sesgos de voluntariedad que podría conllevar la modalidad de "cuestionario online", en donde se pudieron sentir motivados a responder solo quienes se encontraban en situaciones complejas a causa del cambio de modalidad educativa. Y, en torno a las prospectivas de la investigación, es relevante mencionar que el equipo de trabajo se encuentra preparando una segunda versión del estudio, orientada a establecer un contraste entre las conclusiones descritas en el presente artículo y el discurso que pueda manifestar en la actualidad una muestra del profesorado de música chileno. Esto cobra especial relevancia si se considera que en el país se está retornando en forma gradual a modalidades educativas presenciales o semipresenciales.

\section{Referencias}

Angel-Alvarado, R. y Lira-Cerda, J. (2017). Instalaciones y recursos educativos para la educación musical según la representación social de los estudiantes chilenos. Revista Electrónica de LEEME, 40, 19-31. https://doi.org/10.7203/LEEME.40.10412 
Arredondo, C. (2020). Autoridad pedagógica en la educación musical chilena: desde la periferia curricular a la revisión del sistema en medio del estallido social. Átemus, 4(8), 5-13. https://rb.gy/mnomvc

Arriagada, P. (2020). Pandemia Covid-19: educación a distancia. O las distancias en la educación. Revista Internacional De Educación Para La Justicia Social, 9(3e). https://rb.gy/3hfku2

Barraca, J. (2010). Emociones negativas en el profesorado universitario: Burnout, estrés laboral y Mobbing. EduPsykhé: Revista de Psicología y Psicopedagogía, 9(1), 85-100. https://rb.gy/eepgid

Benítez-Corona, L. y Barrón-Tirado, M.C. (2018). Análisis cualitativo de resiliencia en estudiantes de posgrado. Revista Electrónica Educare, 22(1), 125-145. http://dx.doi.org/10.15359/ree.22-1.7

Cisternas, M. (2021). FEDATEB en oposición al retorno presencial a clases: "Emplazamos al ministro a que baje a este territorio a conocer la realidad de cada colegio". Diario Universidad de Chile. https://rb.gy/z5tf8d

Charmaz, K. (2004). Premises, Principles, and Practices in Qualitative Research: Revisiting the Foundations. Qualitative Health Research, 14(7). http://dx.doi.org/10.1177/1049732304266795

Daniec, K. y Torres, C. (2021). La enseñanza de la Música en la virtualidad: reflexiones en torno a la producción de materiales educativos. Revista Iberoamericana de Tecnología en Educación y Educación en Tecnología, 28, 258-265. https://doi.org/10.24215/18509959.28.e32

Domínguez-Lloria, S. y Pino-Juste, M. (2021). La competencia digital en el profesorado de Música de Educación Secundaria en los centros de titularidad pública durante la pandemia derivada de la COVID-19. Revista Electrónica de LEEME, 47, 80-97. https://doi.org/10.7203/LEEME.47.20515

Dussel, I. (2020). La formación docente y los desafíos de la pandemia. Revista Digital EFIDGES, 6(10), 11-25. https://rb.gy/oomdw6

Equipo Elige Educar (12 de enero de 2021). Estudio proyecta que podrían faltar más de 26 mil docentes idóneos para 2025. Elige Educar. https://rb.gy/8gtmlt

Fernández, J., Domínguez, J. y Martínez, P. (2020). De la educación presencial a la educación a distancia en época de pandemia por Covid 19. Experiencias de los docentes. Revista 
Electrónica sobre Cuerpos Académicos y Grupos de Investigación, 7(14), 87-110. https://rb.gy/pmwap2

Fiorentino, M.T. (2008). La construcción de la resiliencia en el mejoramiento de la calidad de vida y la salud. Universidad Nacional de San Luis, Argentina. Suma Psicológica, 15(1), 95-113. https://rb.gy/ezacfx

Grotberg, E.H. (1996). The international resilience project findings from the research and the effectiveness of Interventions. En Annual Convention of the International Council of Psychologists, Banff, Canada. https://rb.gy/z9kcoa

Heiss, C. (2020). Chile: entre el estallido social y la pandemia. Análisis Carolina, 18(1). https://rb.gy/hr9msx

Jiménez-Yañez, C. (2020). \#Chiledespertó: causas del estallido social en Chile. Revista Mexicana de Sociología, 82(4), 949-957. https://doi.org/10.22201/iis.01882503p.2020.4.59213

Jorquera, R., Valverde, X. y Godall, P. (2020). Propuesta de principios alternativos para la educación musical en un contexto latinoamericano. Revista Electrónica de LEEME, 46, 1-16. https://rb.gy/tuyp11

López-Roldán, P. y Fachelli, S. (2015). Metodología de la Investigación Social Cuantitativa. Universidad Autónoma de Barcelona. https://rb.gy/w28mof

Mardones, C. (14 de enero de 2019). Admisión 2019: Disminuye interés por carreras de pedagogía pese a que aumentan las vacantes. El Mercurio. https://rb.gy/s6psj9

Miranda, B., Albert, C. y Sepúlveda, N. (11 de noviembre de 2019). Lesiones oculares: advertencias ignoradas por 20 días podrían ser clave en querella contra Piñera. Centro de Investigación Periodística (CIPER). https://rb.gy/pxeuje

Montoya, J. (2021). Música, Youtuber! La incidencia de Jaime Altozano en las aulas de educación musical. Revista Electrónica de LEEME, 47, 63-75. https://doi.org/10.7203/LEEME.47.19379

Morelato, G. (2011). Resiliencia en el maltrato infantil: aportes para la comprensión de factores desde un modelo ecológico. Revista de Psicología, 29(2), 203-224. https://rb.gy/1xwnkz

Morelato, G. (2014). Evaluación de factores de resiliencia en niños argentinos en condiciones de vulnerabilidad familiar. Universitas Psychologica, 13(4), 1473-1488. https://rb.gy/w48eno 
Muñoz, D. (2020) Encuesta Colegio de Profesores reveló que casi la totalidad de los consultados no aprueba el retorno a clases presenciales. ADN Radio. https://rb.gy/hrkmwc

Noriega, G., Angulo, B. y Angulo, G. (2015). La resiliencia en la educación, la escuela y la vida. Perspectivas Docentes, 58, 42-48. https://rb.gy/letvhc

Olave, J. M., Núñez, M. y Arévalo, A. (2020). Relatos del estallido social en Chile: acontecimiento y formación inicial docente como experiencia narrativa. Márgenes, Revista de Educación de la Universidad de Málaga, 1(3), 272-290. https://doi.org/10.24310/mgnmar.v1i3.9497

Picón, G., González de Caballero, G. y Paredes, J. (2021). Desempeño y formación docente en competencias digitales en clases no presenciales durante la pandemia COVID-19. Arandu UTIC, 8(1), 139-153. https://rb.gy/qgwjm6

Robinet-Serrano, A. y Pérez-Azauanche, M. (2020). Estrés en los docentes en tiempos de pandemia Covid-19. Revista Polo del Conocimiento, 5(12), 637-653. https://rb.gy/bbdmxb

Rodríguez, G., Gil, J. y García, E. (1996). Metodología de la investigación cualitativa. Ed. Aljibe.

Rodríguez-Quiles, J. (2021). Educación musical, epistemocracia y postcovid-19. Revista Electrónica de LEEME, 47, 1-16. https://doi.org/10.7203/LEEME.47.17550

Román, F., Forés i Miravalles, A., Calandri, I., Gautreaux, R., Antúnez, A., Ordehi, D., Calle, L., Poenitz, V., Pérez, K., Torresi, S., Barceló, Ernesto., Conejo, M., Veerle, P. y Allegri, R. (2021). Resiliencia de docentes en distanciamiento social preventivo obligatorio durante la pandemia de COVID-19. Journal of Neuroeducation, 1(2), 72-77. https://doi.org/10.1344/joned.v1i1.31727

del Rincón, D., Arnal, J., Latorre, A. y Antoni, M. (1995). Técnicas de investigación en Ciencias Sociales. Dykinson.

Ruiz, J. (2012). Metodología de la investigación. Universidad de Deusto.

Santander, P. (2011). Por qué y cómo hacer Análisis de Discurso. Cinta de Moebio: Revista Electrónica de Epistemología de Ciencias Sociales, 41, 207-224. http://dx.doi.org/10.4067/S0717-554X2011000200006

Salinas, J., Castellví, J. y Camus, P. (2021). ¡Chile despertó! Una investigación-acción en formación ciudadana de futuros docentes durante el estallido social. Revista Sophia Austral, 26, 325-347. https://rb.gy/spadym

@Raúl Jorquera Rossel, Ximena Valverde Ocariz y Rodrigo Montes Anguita. The content of this article is the sole responsibility of the authors. The Revista Electrónica de LEEME and Universitat de València are not liable for any legal actions that may arise involving the article's content. Revista Electrónica de LEEME - Lista Electrónica Europea de Música en la Educación-. http://ojs.uv.es/index/php/LEEME/index ISSN: 1575-9563. Editores: Universidad de Valencia y Jesús Tejada. Visibilidad de esta revista: SCOPUS, Emerging Sources Citation Index (Clarivate), EBSCO, CINDOC (CSIC), Citefactor, COPAC, Dialnet, DICE (CSIC), DOAJ, e-revistas (CSIC), EBSCO Premier, ERIH+, Gale Cengage Learning, IN-RECS, IRESIE, LATINDEX, MIAR, OCLC Worldcat, RESH, REDIB, RILM Core Journals, SUDOC, ULRICHS. Esta revista es de acceso libre mediante licencia Creative Commons 4.0 CC by. Política de archivado: etiqueta verde SHERPA-ROMEO. 
Silas, J.C. y Vázquez Rodríguez, S. (2020). El docente universitario frente a las tensiones que le plantea la pandemia. Revista Latinoamericana de Estudios Educativos, 50(ESPECIAL), 89-120. https://rb.gy/cnit9s

Silva-Peña, I. y Paz-Maldonado, E. (2019). Formación docente para la justicia social desde la perspectiva emocional: Indagaciones narrativas en el contexto de la revolución del torniquete. Revista Interuniversitaria de Formación del Profesorado, 33(3), 195-212. https://doi.org/10.47553/rifop.v33i3.75652

Strauss, A. y Corbin, J. (2002). Bases de la investigación cualitativa. Técnicas y procedimientos para desarrollar la teoría fundamentada. Universidad de Antioquía.

Tunjo-Guerrero, L. y Yangali, J. (2021). Incidencia de inteligencia creativa del docente en su práctica pedagógica de docentes durante la pandemia. Apuntes Universitarios, 11(3), 241-259. https://doi.org/10.17162/au.v11i3.703

Valverde, X. (2013). Análisis de fortalezas y debilidades en relación al aporte de la Educación Artística en los subsectores de Matemáticas, Lenguaje y Ciencias de los docentes de primer ciclo básico del colegio Hispano Italiano de la ciudad de Iquique. (Tesis de Magíster no publicada). Universidad Andrés Bello.

Valverde, X. (2018). Música tradicional en el aula: las bandas de bronces de Tarapacá y sus aportaciones a la educación musical escolar. (Tesis de Doctorado). Universidad Autónoma de Barcelona. https://rb.gy/z8xhnr

Valverde, X. y Godall, P. (2018). Música tradicional en el aula: aportaciones para un aprendizaje significativo en la escuela. Revista Electrónica de LEEME, 41, 16-34. https://doi.org/10.7203/LEEME.41.10530 